\title{
A Four-Wing Compound Parabolic Concentrator (CPC) Design for Heating and Sanitization of Waste Products
}

\author{
Ababu Teklemariam Tiruneh ${ }^{*}$, William N. Ndlela1, Tendekayi Henry Gadaga ${ }^{1}$, \\ Tesfamariam Debesay ${ }^{2}$, Jonna Heikkilä ${ }^{3}$ \\ ${ }^{1}$ Department of Environmental Health Science, University of Swaziland, Mbabane, Swaziland \\ ${ }^{2}$ Department of Chemistry, University of Swaziland, Kwaluseni, Swaziland \\ ${ }^{3}$ Faculty of Technology Environment and Business, Turku University of Applied Sciences, Turku, Finland \\ Email: *ababute@gmail.com
}

How to cite this paper: Tiruneh, A.T., Ndlela, W.N., Gadaga, T.H., Debesay, T. and Heikkilä, J. (2017) A Four-Wing Compound Parabolic Concentrator (CPC) Design for Heating and Sanitization of Waste Products. Journal of Power and Energy Engineering, 5, 18-35.

https://doi.org/10.4236/jpee.2017.53002

Received: February 21, 2017

Accepted: March 19, 2017

Published: March 22, 2017

Copyright ( $) 2017$ by authors and Scientific Research Publishing Inc. This work is licensed under the Creative Commons Attribution International License (CC BY 4.0).

http://creativecommons.org/licenses/by/4.0/

(c) (i) Open Access

\begin{abstract}
Harnessing the freely available source of energy from the sun offers a number of additional benefits. Not least of these benefits is the fact that solar energy is an environmentally sustainable alternative. A four-wing compound parabolic concentrator (CPC) was designed as a modification of the regular non-imaging CPC concentrator that has a widespread use as solar collector. The design is intended to increase the angle of acceptance as well as concentration of energy from the sun. The conceptual design, mathematical formulation as well as construction and initial trial results have been presented in this paper. Pilot trials of the four-wing concentrator used for sanitizing both liquid and waste products produced satisfactory results. Improvements in terms of design as well as material used for construction and better preservation of heat can be considered further in the future research.
\end{abstract}

\section{Keywords}

Solar Concentrator, Compound Parabolic Concentrator, Sanitization, Sterilization, CPC, Non-Imaging Concentrators,

Parabolic Concentrators, Waste Drying

\section{Introduction}

The energy from the sun is vast, while being commonly available at any point of use. So far only a small proportion of solar energy is being used even in the present age of advanced technology. Solar concentrators are one of the devices used for concentrating and using solar energy for various purposes including water heating, distillation, concentration of solids and sterilization to name a few. 
The two major classifications of solar collectors are namely imaging and nonimaging types [1]. The compound parabolic concentrator is a non-imaging type concentrator that is able to concentrate rays from the sun from wider angles to smaller absorber surface without the need for tracking the sun. Although the sun ray falling on the absorber is not focused, there is considerable concentration of sun ray concentration that can be attained with a CPC design. Specialized designs for ultra-concentration of sun rays involving second stage concentration have been developed for applications such as complete degradation of wastes [2].

The origin of the CPC type of collectors dates back to the 1960s [3]. Since then CPC profiles have been used worldwide for wide range of applications including heating, sterilization, photo electric cell applications, etc. The initial descriptions of CPC were given as three dimensional CPC by Baranaov [4] [5] and Baranov and Melnikov [6]; as axially symmetric CPC by Ploke [7] including refraction elements in the design. The thermodynamic limit to concentration was noted by Hinterberger and Winston [8] [9]. The theoretical basis for the thermodynamic limit was provided by Winston [10]. Smestad et al. [11] also proved that the same thermodynamic limit applies for the CPC design using the Edge Ray principle. This also meant that the compound parabolic concentrator has the maximum concentration ratio. Two dimensional CPC profiles and formulas for calculating the dimensions have been described in elaborate detail by Winston and Hinterberger [12]. Harper et al. [13] applied three-dimensional CPC to infrared collection.

The concentration ratio of compound parabolic concentrators (CPC) is the ratio of the width of the aperture at the top to that of the width of the absorber at the bottom. Concentration ratios of CPCs normally range from 3 to 10 [1]. It will be shown later using Fermat's principle that the maximum concentration ratio for the $\mathrm{CPC}$ is given by:

$$
C_{R}=\frac{W}{b}=\frac{1}{\operatorname{Sin}\left(\theta_{c}\right)}
$$

where $C_{R}$ is the concentration ratio, $W$ is the aperture width, b is the absorber width and $\theta_{c}$ is the half acceptance angle. The acceptance angle $2 \theta_{c}$ is the maximum angular opening that will allow the radiation from the sun to be trapped through the aperture width $W$ and get concentrated on the absorber width $b$. From the formula for concentration ratio it is apparent that for a given angle of acceptance there is a limit to the maximum concentration ratio as given by Equation (1).

The limit to concentration is also supported by the Second Law of Thermodynamics which states that heat cannot spontaneously flow from a colder body to a hotter body. Stated in another way the natural path of heat transfer is always in the direction of increasing entropy. This limit to concentration from thermodynamics perspective was explained well by A. Rabl [14]. Rabl also derived the mathematical formulation for CPC design including reflector shape, height, length, width and arc length [15]. The limit to concentration ratio without violating the Second law of thermodynamics is shown to be the same as the one 
given by Equation (1) above.

The limit to concentration can also be derived by the Edge Ray Principle using Fermat's theory which states that light travels between two points using the path that results in minimum time of travel [16]. This means that in Figure 1, rays approaching the CPC profile in parallel at $A$ and $C$ will arrive at the same time at the absorber $B^{B}$ which is the focal point of the reflector. Since both rays should take minimum time while starting from similar positions and travelling with the speed of light, they should, therefore, travel equal distances. This means that the paths $C A B^{\prime}$ and $A^{\prime} B B^{\prime}$ should be of equal distance.

Using the geometry of angle of acceptance $\theta$ shown in Figure 1, it can be easily shown that the concentration ratio is equal to:

$$
C_{R}=\frac{A A^{\prime}}{B B^{\prime}}=\frac{1}{\operatorname{Sin}(\theta)}
$$

The Edge Ray Principle also led to String Method of constructing the reflector profile which can be applied to a wide range of reflector and absorber profiles. In the String Method, given the focal point of absorption and the starting point of the ray along the edge ray wave front (Figure 1), the constant string length can be used to draw the reflector profile starting from point $A$ and ending at point $\mathrm{B}$ in Figure 1. At any point $X$ generated as part of the profile during the drawing, the constant length of the string is stretched between points $C, B$ and the point $X$ being plotted.

Various design modifications to the CPC have been proposed over the years in order to optimize their performance. For example the upper portion of the $\mathrm{CPC}$ reflector can be truncated without appreciably reducing the concentration ratio since doing so will not reduce the aperture width significantly [17]. Such a measure will be helpful in reducing materials cost as well as increasing the angle of acceptance through a truncated profile. Asymmetric designs involving two different parabolas of different focal length are combined in order to increase the angle of acceptance and allow more diffuse ray into the absorber [18]. Rönnelid

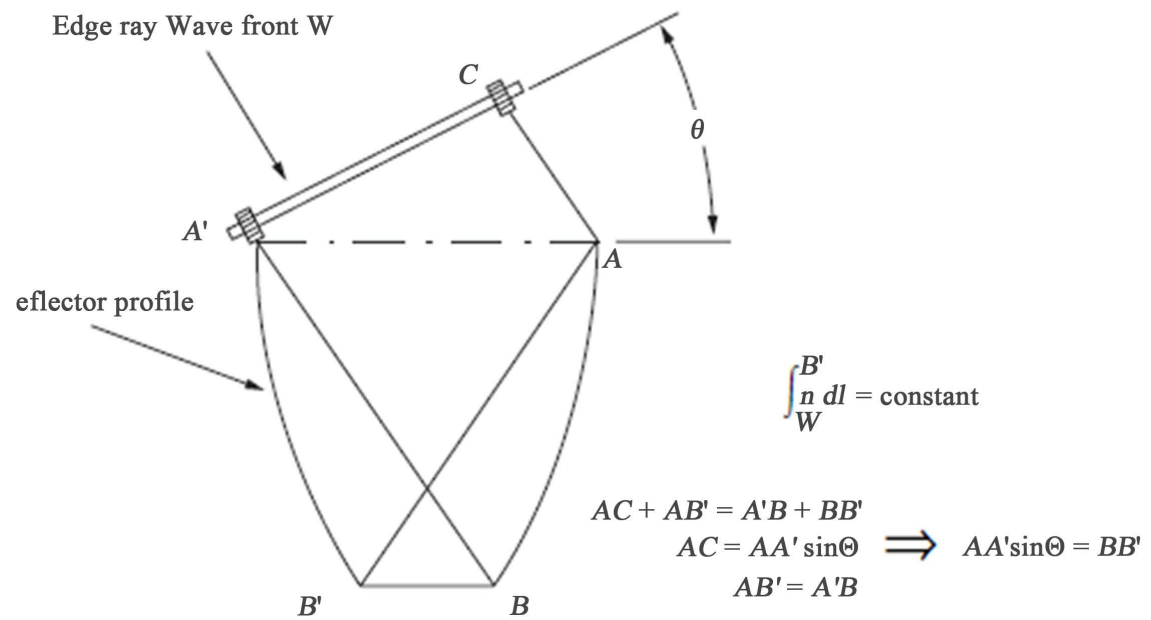

Figure 1. The concentration ratio and String method as derived by the Edge Ray principle. 
and Karlsson [19] designed a truncated asymmetric CPC generated from otherwise symmetric parabolas having the same focal length while at the same time sharing the same position of the focal point. However, the truncation is made through a line that is not perpendicular to the axis of symmetry thus generating asymmetry in the process. The objective is to collect as much of the diffuse ray as possible.

Even though the extent of concentration of solar rays by CPCs is limited, the stationary nature of CPC means that there is less need for tracking the sun. Compound parabolic concentrators are, therefore, convenient for stationary household applications such as for providing hot water or photo voltaic cells [20].

Receiver materials intended for CPC should have higher heat absorbance as well as being heat conductive to allow heat to be transferred to the receiving medium [1]. Surface coatings such as silicon polymer are used to increase absorbance. To preserve heat in CPC, top covers are often provided. The cover should be transparent while being durable and low cost. Reflectors should also be carefully selected and should have high reflectance values. Alternatively, reflector layer such as silver tint can be applied to increase the reflectance of the surface.

The temperature range that can be generated from compound parabolic concentrators depends on the design provision made for limiting heat losses by conduction and convection. Concentrators without non-evacuated tubes work in the temperature range of $80^{\circ} \mathrm{C}-100^{\circ} \mathrm{C}$. On the other hand, concentrators with evacuated tubes have the air inside the tube removed. The creation of partial vacuum limits the loss of heat by conduction and convection. Such concentrators can achieve working temperature in the $100^{\circ} \mathrm{C}-200^{\circ} \mathrm{C}$ range.

\section{Materials and Methods}

The methodological approach of the development of the four wing compound parabolic collector design is presented below including the mathematical formulas used for computing the shape dimensions as well as concentration ratios. The four-wing CPC, shown in Figure 2, consists of the complete sections of the two identical parabolas constructed together. It is recalled that the regular CPC consists of two wings that are joined together by truncating the portions of the parabola lying on the outer sides of the focal points. In the design presented in this research, instead of truncating these portions, they are included and are made to contribute to the trapping of sun rays thereby increasing both the angle of acceptance and the concentration ratios.

According to this design, during the early hours of the day, the right most wing of the parabola reflects to the outside of the absorber (Figure 2). Later in the day and before noon time, both branches of the left wing parabola reflect rays to the absorber, one to the inside and the other branch to the outside of the absorber. When the sun is overhead the setup works as the regular CPC and as the sun ray progresses to the afternoons, the reflection task switches to the other right wing section of the parabola mirroring the function described for the leftwing parabola. 


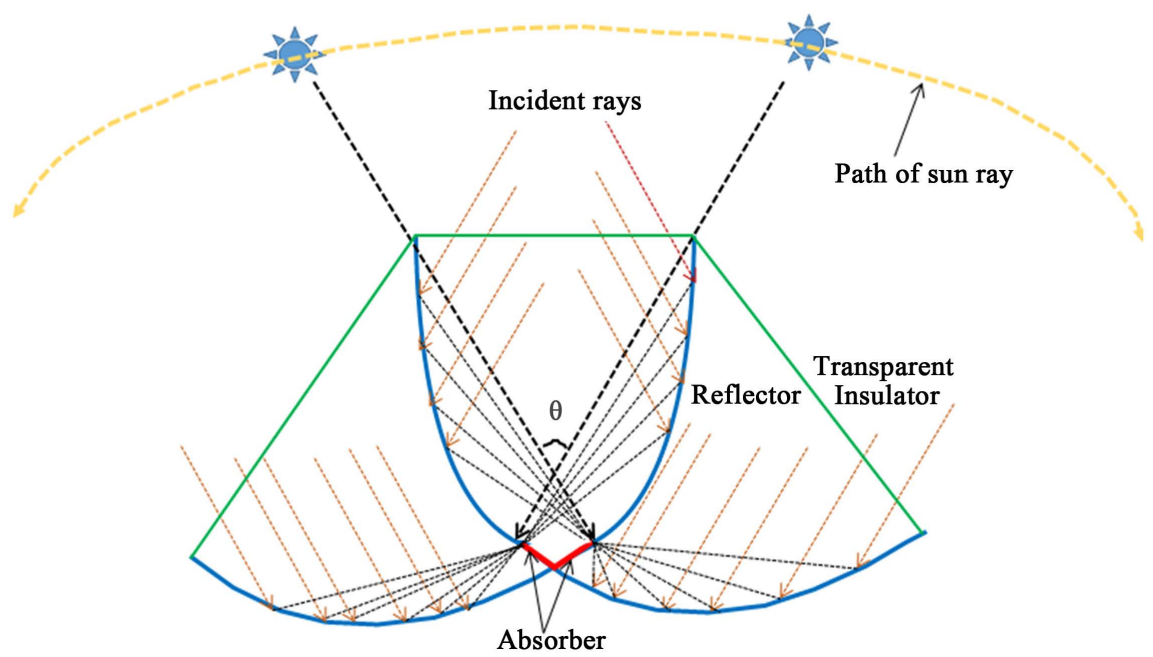

Figure 2. Profile sketch of a four wing compound parabolic collector with v-shaped absorber.

In addition to reflection of direct rays on to the absorber, there are also some secondary rays that will be trapped between the outer and inner surfaces of the two different parabolas as shown in Figure 2 and Figure 3. These reflected rays are useful when some of the reflected rays do not fall directly on the absorber and instead fall on the surface of the adjoining parabola. For compound parabolic concentrators this happens when rays-whose angle of incidence is less than the angle of incidence of the rays parallel to the axis of the parabola-are falling on the reflector. In this case, the reflected rays will not be trapped and directed to the absorber. Instead they escape back to the atmosphere.

The schematic layouts of two possible alternative configurations of the absorber for the four wing CPC design are shown in Figure 2 and Figure 3. These two profiles are more or less similar in their reflector configurations. However they differ in the shape of the reflector, the way the absorber shape is provided, the relative position of the reflector branches and the number and positions of the focal points. In the first design shown in Figure 2, the absorber is $V$-shaped while the second design shown in Figure 3 has a flat absorber profile. The profile with the flat absorber has common focal point at the center for the internal wings while the focal points for the outer wings are located on the left and right edges of the absorber. The outer wings are also shifted outward in both directions which shifts their focal point from the center towards the edges of the absorber.

The v-shaped absorber shown in Figure 2 is slotted at the center which creates a gap at the intersection of the two parabolas. On this portion that the $V$-shaped absorber seats on, the reflector material will not be constructed. Instead only the frame holding the reflector sheet metal is extended to provide continuity to each of the left and right wing complete section parabolic profiles.

In the flat plate horizontal absorber configuration shown in Figure 3, the focal points of the two internal parabolic mirrors are set to coincide at the center of the absorber. Therefore, the internal reflectors share the same focal point. This 


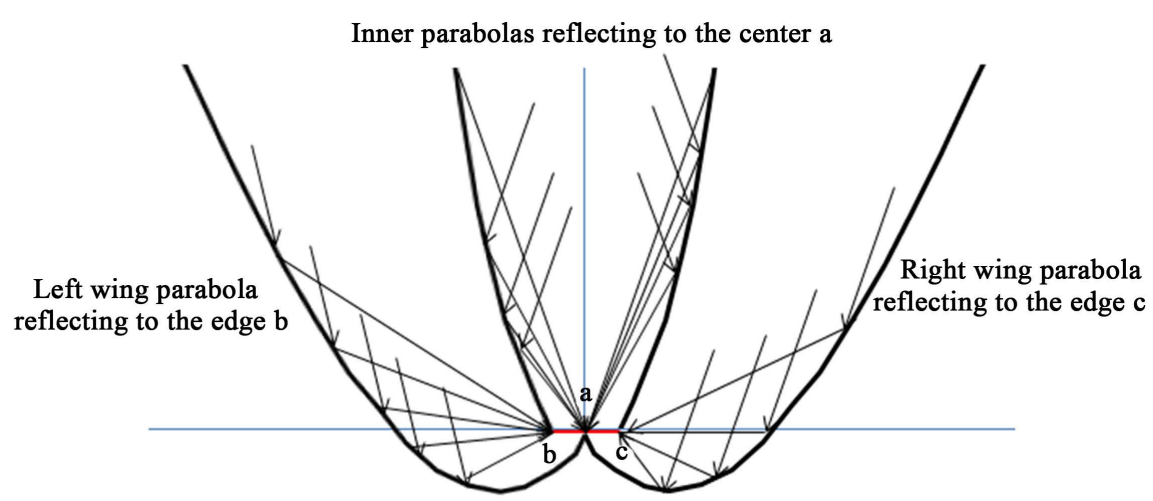

Figure 3. Alternative profile of compound parabolic concentrator with flat-base absorber.

approach obviously reduces the angle of acceptance of the internal wings. However, this is partly offset by the greater angle of acceptance of the outer wings. The angles of acceptance of the inner and outer wings are inversely related. When the interior reflector wings have their angle of acceptance reduced, the outer wings will have their angle of acceptance increased and vice versa.

For the flat plate absorber configuration, in order to ensure continuity of reflected rays' path to the absorber, the outer wings are displaced inwards such that they intersect at the center of the absorber as shown in Figure 3. Because of the inward translation, the outer wings will have their focal point shifted towards the edges of the absorber. The flat plate absorber configuration has, therefore, three foci all of which are located on the absorber plate as shown in Figure 3. Point $\mathrm{a}$ is the focal point which is common to the two internal wings. Point $\mathrm{b}$ is the focus of the outer leftmost wing while point $c$ is the focal point of the outer right most wing.

\subsection{Computation of Reflector Profiles}

The procedure for computation of the reflector profile is explained below for the constructed v-shaped absorber. However, similar procedure can be followed for the flat-plate absorber profile modified for the shapes and the three different focal points $a, b, c$ as shown in Figure 3.

While there are a number of different approaches available for calculating the profiles of the compound parabolic concentrators, transformation of coordinates is used in this research paper for relating the coordinates $(x, y)$ before tilting with the final positions $\left(x^{\prime}, y^{\prime}\right)$ after tilting by an angle of $\theta$ degrees as shown in Figure 4. For Cartesian coordinates, the counter clockwise angle coordinate transformation equations are given by:

$$
\begin{array}{r}
X=X^{\prime} \operatorname{Cos}(\theta)-Y^{\prime} \operatorname{Sin}(\theta) \\
Y=X^{\prime} \operatorname{Sin}(\theta)+Y^{\prime} \operatorname{Cos}(\theta)
\end{array}
$$

The reverse transformations of the clockwise angle are obtained by substituting $(-\theta)$ in Equations (3) and Equation (4) above for $\theta$;

$$
X^{\prime}=X \operatorname{Cos}(\theta)+Y \operatorname{Sin}(\theta)
$$




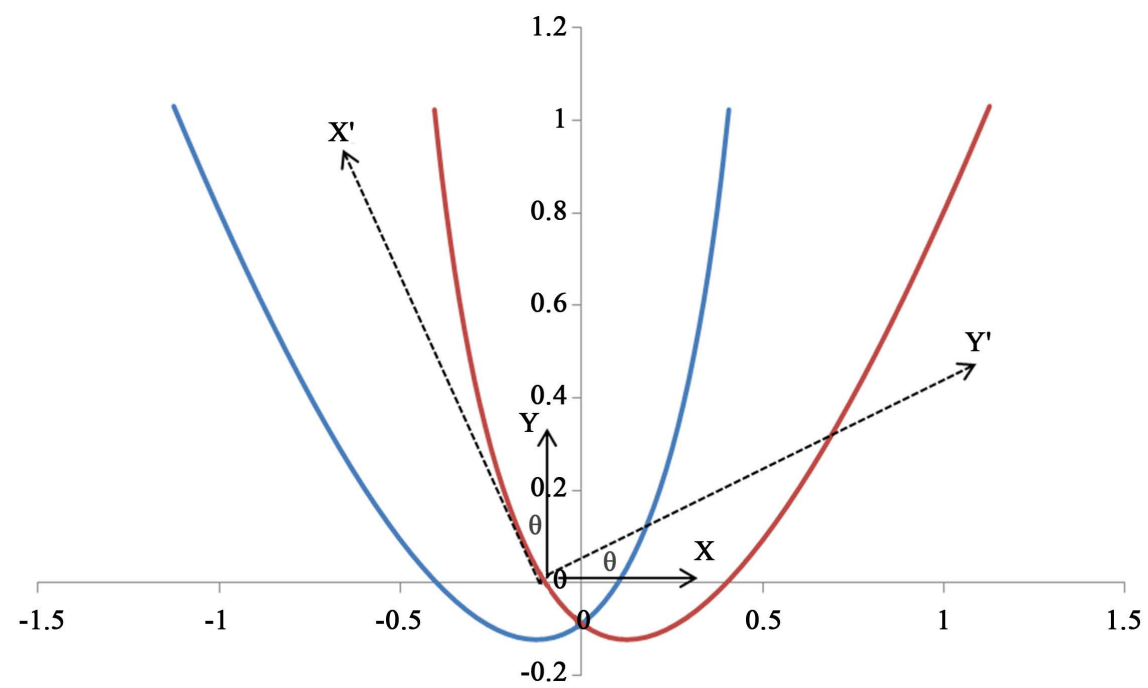

Figure 4. The four wing CPC profile position with respect to normal and tilted coordinates.

$$
Y^{\prime}=-X \operatorname{Sin}(\theta)+Y \operatorname{Cos}(\theta)
$$

The equation of the parabola in the tilted coordinates using the left side of the focus as the origin of coordinates in Figure 4 is given by

$$
Y^{\prime}=\frac{X^{\prime 2}}{4 f}-f
$$

where $f$ is the focal length of the parabola.

To relate the focal length with the horizontal length of the absorber width, first the left and right coordinates of the absorber width are set to coincide with the left and right focal points of the parabola; these points are given by:

Left coordinates of the focal point: $\left(x_{l}, y_{l}\right)=(0,0)$

Right coordinates of the focal points $\left(x_{r}, y_{r}\right)=(b, 0)$

Using the transformation of coordinates from $(x, y)$ to $\left(x^{\prime}, y^{\prime}\right)$ in Figure 4 above the coordinates of the two focal points are given by;

Left coordinates of the focal point: $\left(x_{l}^{\prime}, y_{l}^{\prime}\right)=(0,0)$

Right coordinates of the focal points $\left(x_{r}^{\prime}, y_{r}^{\prime}\right)=[b \cos (\theta),-b \sin (\theta)]$

Substituting the $X^{\prime}$ and $Y^{\prime}$ coordinates of the right focal point in the equation of the parabola of Equation (7), yields:

$$
\begin{gathered}
\frac{(b \cos (\theta))^{2}}{4 f}-f=-b \sin (\theta) \\
4 f^{2}-4 f b \sin (\theta)-b^{2} \cos ^{2}(\theta)=0 \\
f=\frac{4 b \sin (\theta)+\sqrt{16 b^{2} \sin ^{2}(\theta)+16 b^{2} \cos ^{2}(\theta)}}{2 * 4}
\end{gathered}
$$

Simplifying the above expression gives the focal length $f$ in terms of absorber width $b$

$$
f=\frac{b}{2}[(1+\sin (\theta))]
$$


The height of the full length of the tilted parabolas is calculated from the geometric relation shown in Figure 5:

$$
\begin{gathered}
\operatorname{Tan}(\theta)=\frac{\frac{W+b}{2}}{H}=\frac{W+b}{2 H} \\
H=\frac{W+b}{2 \operatorname{Tan}(\theta)}=\frac{W(1+b / W)}{2 \operatorname{Tan}(\theta)}=\frac{W(1+\sin (\theta))}{2 \operatorname{Tan}(\theta)}
\end{gathered}
$$

In the above derivation, use is made of the inverse of the concentration ratio, i.e., $b / W=\sin (\theta)$. Therefore the full height of the parabola is expressed as:

$$
H=\frac{W(1+\sin (\theta))}{2 \operatorname{Tan}(\theta)}
$$

For the overall concentration of the four wing CPC, the total width of concentration includes the width of the aperture corresponding to either of the tilted parabolas as shown in Figure 6.

The maximum total width of the aperture of the four wing CPC is the sum of the internal width $W$ considered above and the additional external aperture width due to the added external wing, $W_{E}$.

$$
W_{\max }=W+W_{E} ; C_{R \max }=\frac{W+W_{E}}{b}
$$

The minimum width of aperture corresponds to rays falling on the right wing of the parabola when the incident angle of the rays is greater than the rays shown above that are parallel to the axis of the parabola. During this time the inner parabolas do not trap the rays. This minimum width corresponds to $W_{E}$.

$$
W_{\min }=W_{E} ; C_{R \min }=\frac{W_{E}}{b}
$$

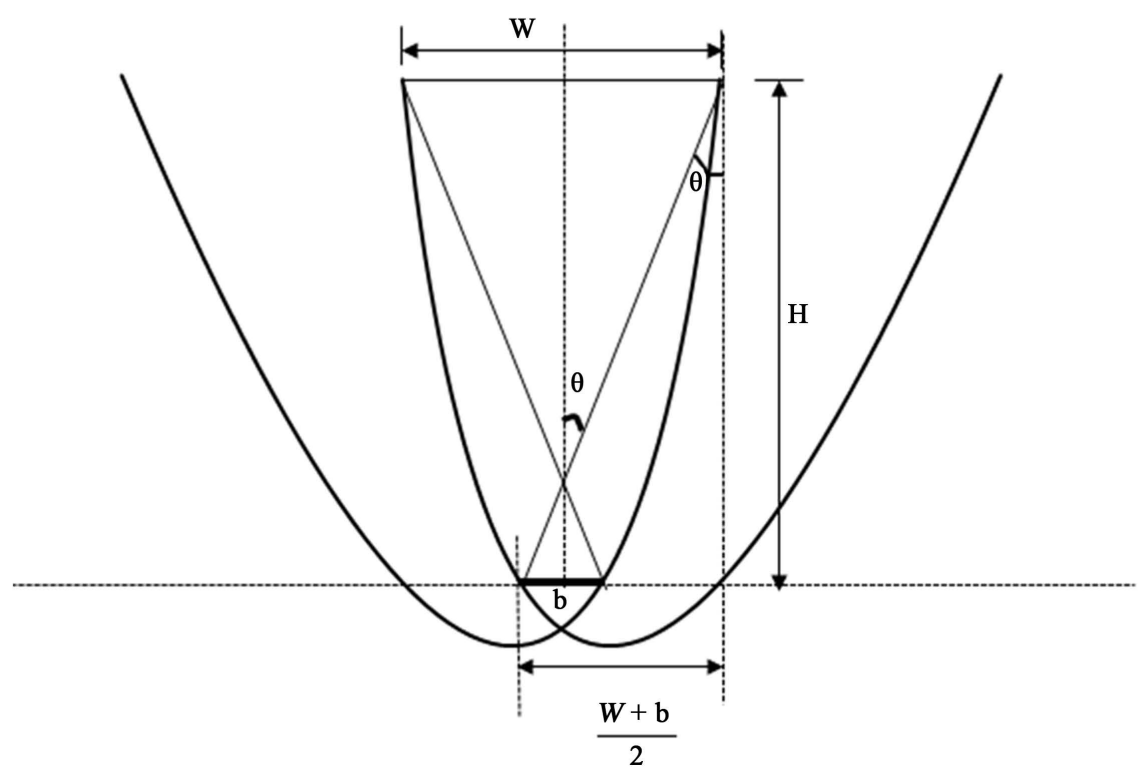

Figure 5. Dimensions of the internal wings of the regular CPC. 


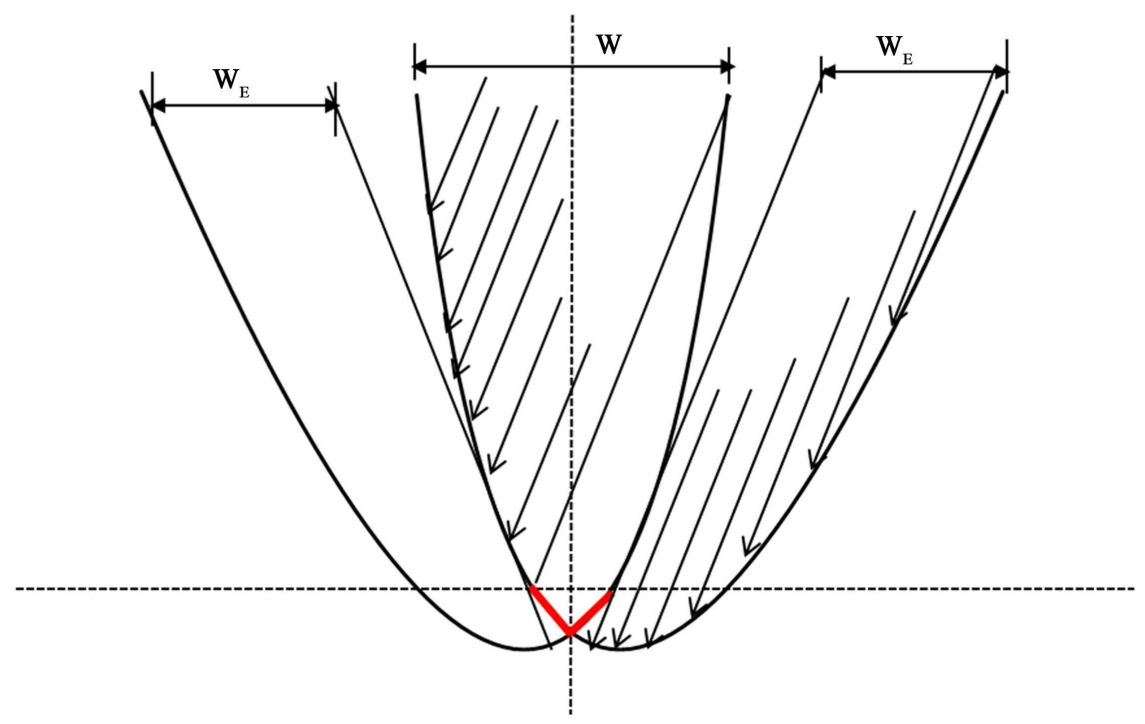

Figure 6. Additional aperture widths $\left(W_{E}\right)$ due to the added outer wings of the parabola.

In between these two minimum and maximum value, there occurs the average concentration of the rays within the angle of acceptance of the inner parabolas corresponding to the aperture width of $W$ and absorber width $b$, i.e.,

$$
W_{\text {average }}=W ; C_{R \text { average }}=\frac{W}{b}
$$

The absorber width depends on the configuration of the absorber. If the absorber is $V$ shaped the full length of the $V$ shape absorber shall be used which will give lesser concentration ratio. On the other hand if the absorber is flat, the flat width $\mathrm{b}$ of the absorber is used in calculating the concentration ratio.

The actual range of concentration ratio as used in the construction has been calculated and will be described in detail in the results section. Since the upper portion of the parabola has limited contribution to the upper width, it is often helpful to truncate the upper portion in order to save material while at the same time increasing the angle of acceptance because of the truncation. The constructed four wing compound parabolic concentrator has, therefore, taken this advantage by limiting the vertical height to 1.0 meter only. This is also given in detail in the results section.

\subsubsection{Length of the Reflectors}

The total length of the reflector profiles for the purpose of determining the material requirement has been obtained by mathematical integration of the entire parabolic profile. For the given equation of the parabolic reflector in terms of the tilted coordinates (Equation (7));

$$
Y^{\prime}=\frac{X^{\prime 2}}{4 f}-f
$$

The total length of the parabolic profile is calculated from the line integral:

$$
S=\int\left(1+\left(\frac{\mathrm{d} Y^{\prime}}{\mathrm{d} X^{\prime}}\right)^{2}\right)^{1 / 2} \mathrm{~d} x=\int\left(1+\left(\frac{X^{\prime}}{2 f}\right)^{2}\right)^{1 / 2} \mathrm{~d} x
$$


The solution for this integral evaluated between the ranges of the $X^{\prime}$ coordinates is given by:

$$
S=f\left[\frac{X^{\prime}}{2 f} \sqrt{1+\left(\frac{X^{\prime}}{2 f}\right)^{2}}+\ln \left(\frac{X^{\prime}}{2 f}+\sqrt{1+\left(\frac{X^{\prime}}{2 f}\right)^{2}}\right)\right]
$$

The focal length is determined from the horizontal width of the absorber and angle of acceptance that is chosen for the design. The focal length of Equation8 was given as:

$$
f=\frac{b}{2}[(1+\sin (\theta))]
$$

Once the horizontal boundary limits of the reflector profiles are determined after truncation of the less useful upper portion of the reflector, the total length of the reflector is computed using Equation (12). The detail of this workout for the constructed CPC concentrator is explained in the results section.

\subsubsection{Determination of Fecal Coliform Counts}

The fecal coliform counts in composted fecal matter, fresh fecal matter, stored urine and grey water samples were determined to assess the effectiveness of sanitization using the CPC. The 3 tube Most Probable Number (MPN) method using Brilliant Green Lactose Bile Broth (BGLBB) was used [21]. Briefly, three consecutive serial dilutions $\left(10^{-1}, 10^{-2}, 10^{-3}\right)(1 \mathrm{~mL})$ of each sample were inoculated into test tubes containing BGBB $(9 \mathrm{~mL})$ and a Durham tube, and were incubated at $44.5^{\circ} \mathrm{C}$ for $24 \mathrm{~h}$. Presumptive positive tubes were shown by growth and gas production. The most probable number (MPN) of coliforms was estimated from the number of tubes inoculated and the number of positive tubes using statistical tables [22].

\section{Results and Discussion}

\subsection{Drawing and Specification of the Four Wing Compound Parabolic Concentrator}

The vertical height of the reflector was limited to 1.0 meter by truncating the less useful upper portion in order to save material since its contribution to increasing the concentration ratio is limited owing to the limited aperture width it contributes. On the other hand truncation of the upper portion results in greater angle of acceptance of the rays both for the inner as well as the outer wings of the parabola.

The horizontal dimensions of the reflector after truncation is specified to be approximately $1.12 \mathrm{~m}$ on both side of the center of the reflector giving a total horizontal width of $2.24 \mathrm{~m}$ for the entire concentrator setup. The total height of the reflector including the $0.12 \mathrm{~m}$ vertical section below the top of the absorber for the outer wings is 1.12 meter. These horizontal dimensions are specified after the origin of coordinates was shifted from the left focal point to coincide with the vertical central axis of the reflector so that the left and right wings of the reflectors are mirror images of each other. In this way it is enough to specify the 
dimension of one side of the reflector and construct the other wings using the same dimension on the opposite side of the central vertical axis.

The dimension specifications in terms of the normal $x$ and $y$ coordinates are worked out using the transformation Equations (3)-(6) within the above stated horizontal and vertical boundary limits. The coordinate specifications are tabulated in Table 1. Figure 7 shows the reflector profile for the chosen four wing CPC after the coordinates are plotted on the $x$ - $y$ axis.

The total length of each of the complete reflector profile (the left wing and right wings together) was calculated using Equation (12). According to this equation, the integral expressed is given by.

$$
S=f\left[\frac{X^{\prime}}{2 f} \sqrt{1+\left(\frac{X^{\prime}}{2 f}\right)^{2}}+\ln \left(\frac{X^{\prime}}{2 f}+\sqrt{1+\left(\frac{X^{\prime}}{2 f}\right)^{2}}\right)\right]
$$

The limits of the tilted coordinates corresponding to the shifted normal $\mathrm{x}$ coordinates are worked out by the Catrtesian coordinate transformation. Accordingly, the mapping of the coordinates are as follows:

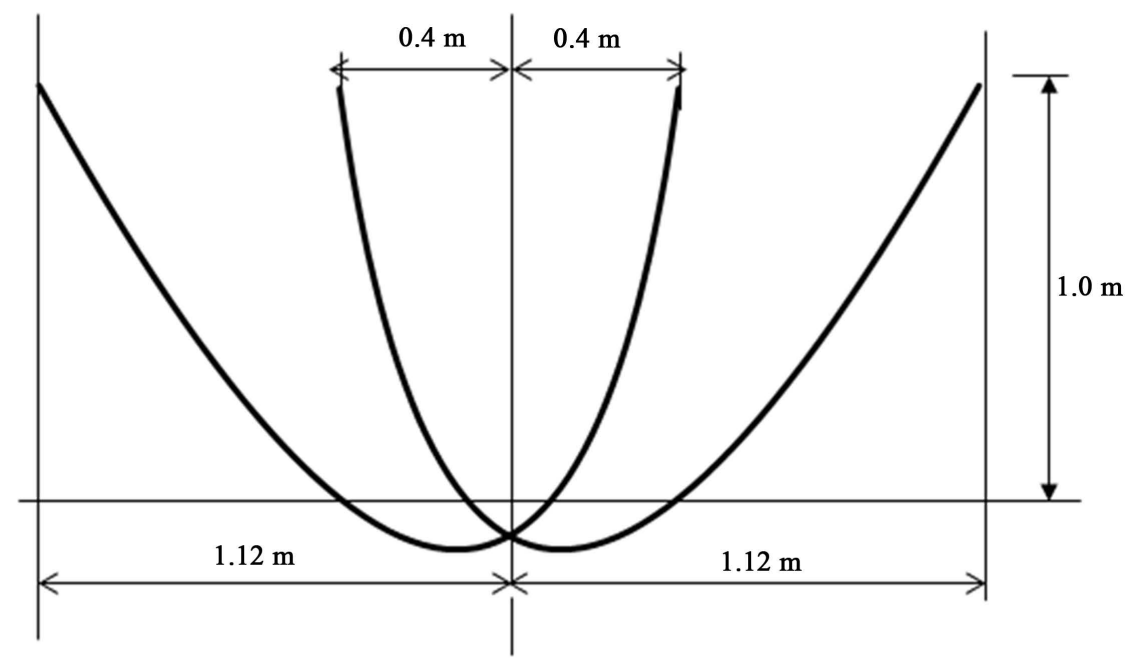

Figure 7. Reflector profile chosen for construction of the four wing CPC concentrator.

Table 1. Dimensions of the four wing CPC measured from the from the top midpoint of the absorber.

\begin{tabular}{cccccccc}
\hline$X(\mathrm{~m})$ & $Y(\mathrm{~m})$ & $X(\mathrm{~m})$ & $Y(\mathrm{~m})$ & $X(\mathrm{~m})$ & $Y(\mathrm{~m})$ & $X(\mathrm{~m})$ & $Y(\mathrm{~m})$ \\
\hline-1.126 & 1.030 & -0.535 & 0.130 & -0.076 & -0.118 & 0.249 & 0.289 \\
-1.045 & 0.882 & -0.470 & 0.063 & -0.028 & -0.102 & 0.281 & 0.386 \\
-0.966 & 0.744 & -0.407 & 0.006 & 0.018 & -0.077 & 0.310 & 0.493 \\
-0.889 & 0.616 & -0.347 & -0.040 & 0.062 & -0.042 & 0.337 & 0.610 \\
-0.814 & 0.498 & -0.289 & -0.076 & 0.103 & 0.004 & 0.362 & 0.737 \\
-0.741 & 0.391 & -0.232 & -0.101 & 0.143 & 0.060 & 0.385 & 0.875 \\
-0.670 & 0.293 & -0.178 & -0.117 & 0.180 & 0.126 & 0.406 & 1.023 \\
-0.601 & 0.206 & -0.126 & -0.122 & 0.216 & 0.202 & & \\
\hline
\end{tabular}




$$
\begin{gathered}
\left(X_{\text {left }}, Y_{\text {right }}\right) \rightarrow\left(X_{\text {left }}^{\prime}, Y_{\text {right }}^{\prime}\right) \\
(-1.12,+0.4) \rightarrow(-0.8,+0.7)
\end{gathered}
$$

The total length of the profile for the entire concentrator, according to Equation (12) of the integral expression is caculated by substituting the above bounday conditions for $\mathrm{x}$ values and multiplying the result by two for the two wing prfiles.

Total length of profile $=2.917 * 2=5.834$ meters

Each of the right and left wings of the concetratrator reflectors have a profie length of 2.92 meters.

The acceptance half-angle used for generating the profile is $11.5^{\circ}$. This results in a total angle of acceptane of $23^{\circ}$. It is noted that increasing the angle of acceptance to trap more ray from the sun would result in decrease of the concentration ratio since the absorber width will also increase.

\subsection{Construction of the Four Wing CPC Concentrator}

The reflector surfaceswereconstructed from galvanised iron sheet metal of $1 \mathrm{~mm}$ thickness. This metal surface has, on its own, surface reflection chracteristics. However, for better reflection a silver tint was applied on all the surfaces of the reflector including the outer surfaces of the inner wings to enable refelection of secondary rays towards the absorber.

The absorber material chosen was mild steel of $3 \mathrm{~mm}$ thickness. It was bent into the required $\mathrm{V}$ shape so that the top width has a dimension of $20 \mathrm{~cm}$ and the side lengths are $14 \mathrm{~cm}$ long on each side. For better absorption, the absorber is painted black on both the outside and inside surfaces as both surfacesreceive rays from the sun. The absorber and reflected edges are set to meet in seamless fashion so that there is continuity of the path of the reflected rays towards the absorber.

The dimesnisons were initially setup on flat metal and by using flat bars the necessary curvature of the reflector was established as shown in Figure 8.

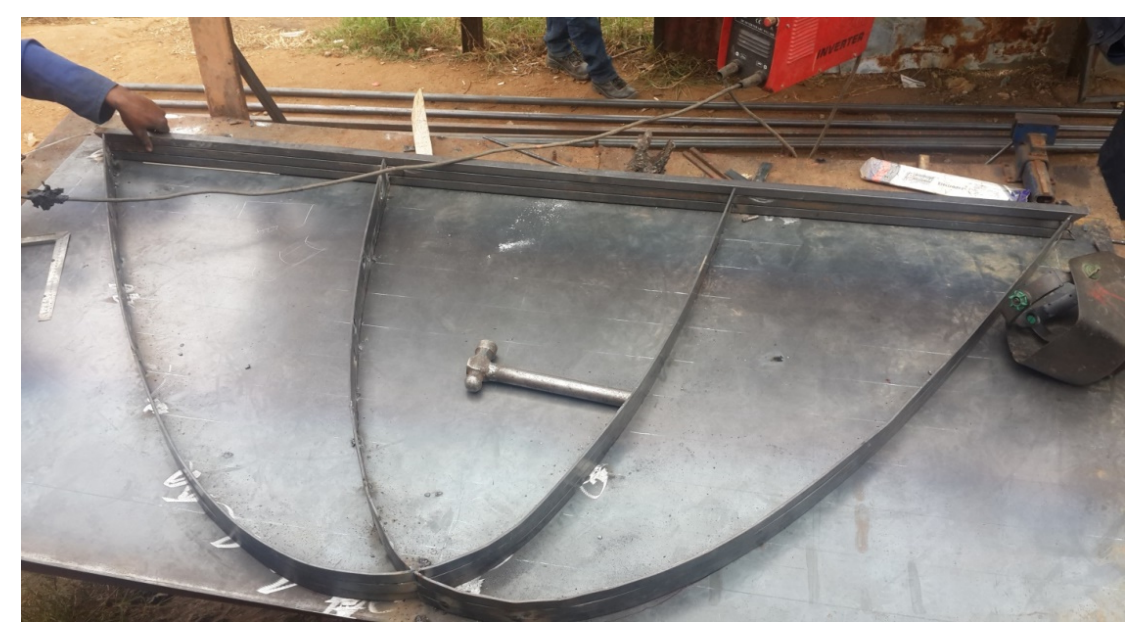

Figure 8. Constructing the four wing CPC profile on a sheet metal base. 
The frames of the reflector surfaces were constructed using a combination of 5 $\mathrm{cm}$ flat bars and $12 \mathrm{~mm}$ reinforcement bars made in such away that the shape is maintained and the profile would be rigid and would not change due to stresses during transportation and use.

The entire concentrator setup was mounted on a rectangular frame seater constructed in such away that it is possible to adjust the tilting and orientation of the concentrator as needed and whenever sun tracking is required. This is shown in Figure 9.

In order to preserve the heat that is trapped, the top surface roof of the reflector was covered with $4 \mathrm{~mm}$ Perspex glass. The side walls of the reflector were covered with polyethylene sheets in order to preserve the heat generated during use. These are shown in Figure 10 and Figure 11.

Finally the setup was installed at the pilot research center in Mnyamatsini for trial at a specific location where the concentrator is fully exposed to sunray throughout the day. Figure 12 shows the CPC installed for trial and use.

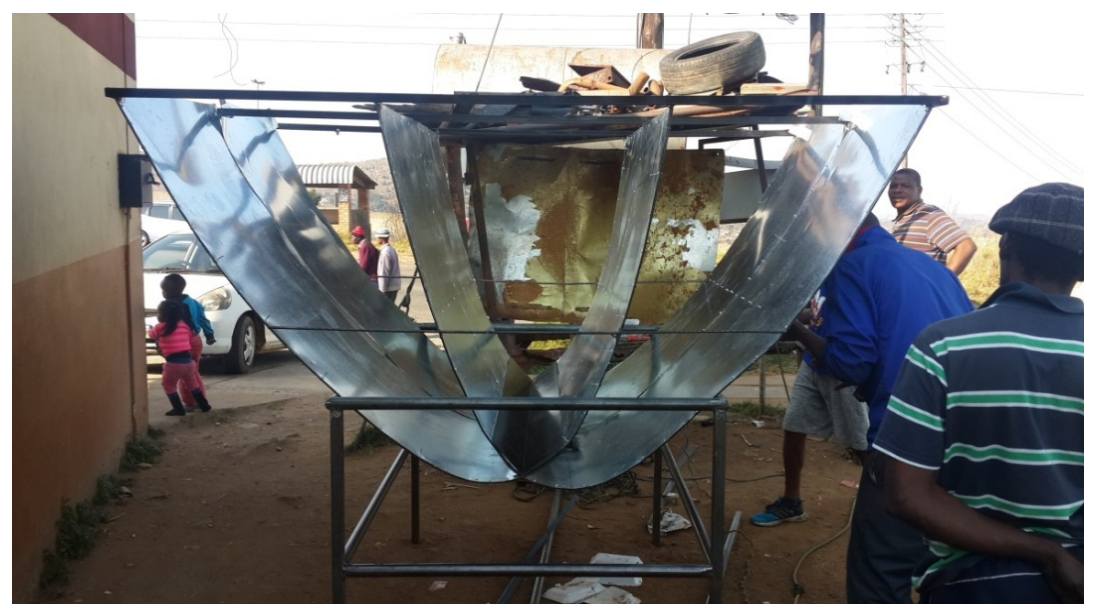

Figure 9. Construction of the wings, frame, absorber and reflector surfaces.

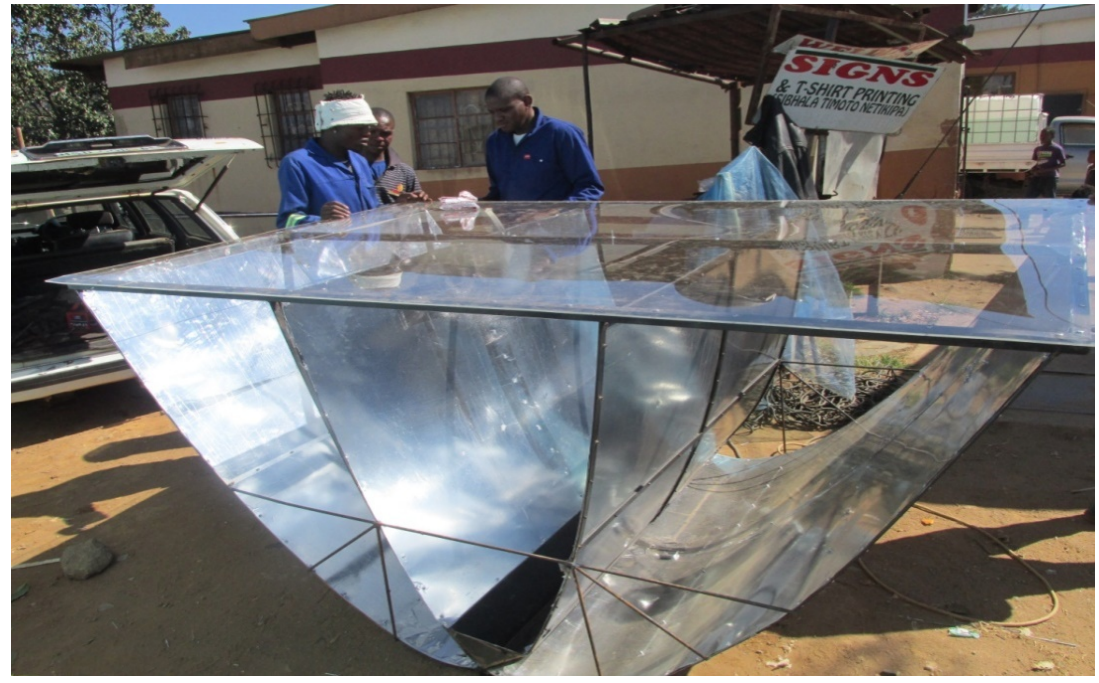

Figure 10. Top Perspex glass cover of $4 \mathrm{~mm}$ thickness fitted for insulation and preservation of heat. 


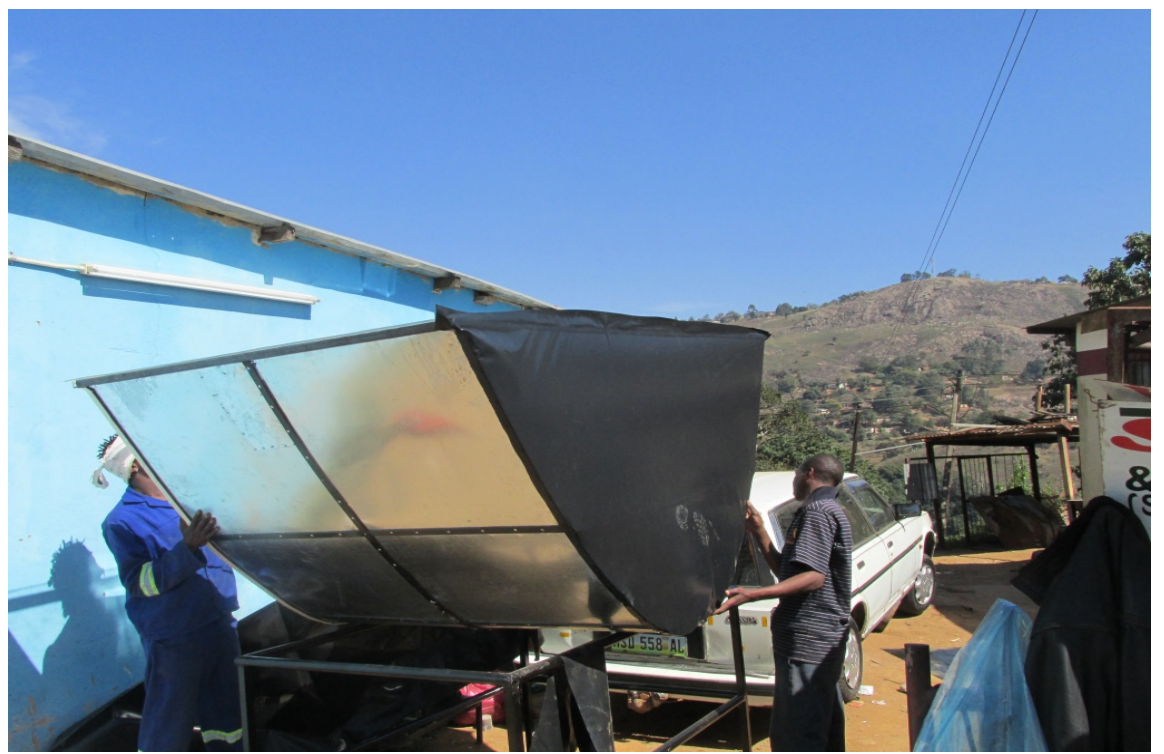

Figure 11. Side walls of the four wing CPC collectors wrapped with polyethylene sheets to preserve heat.

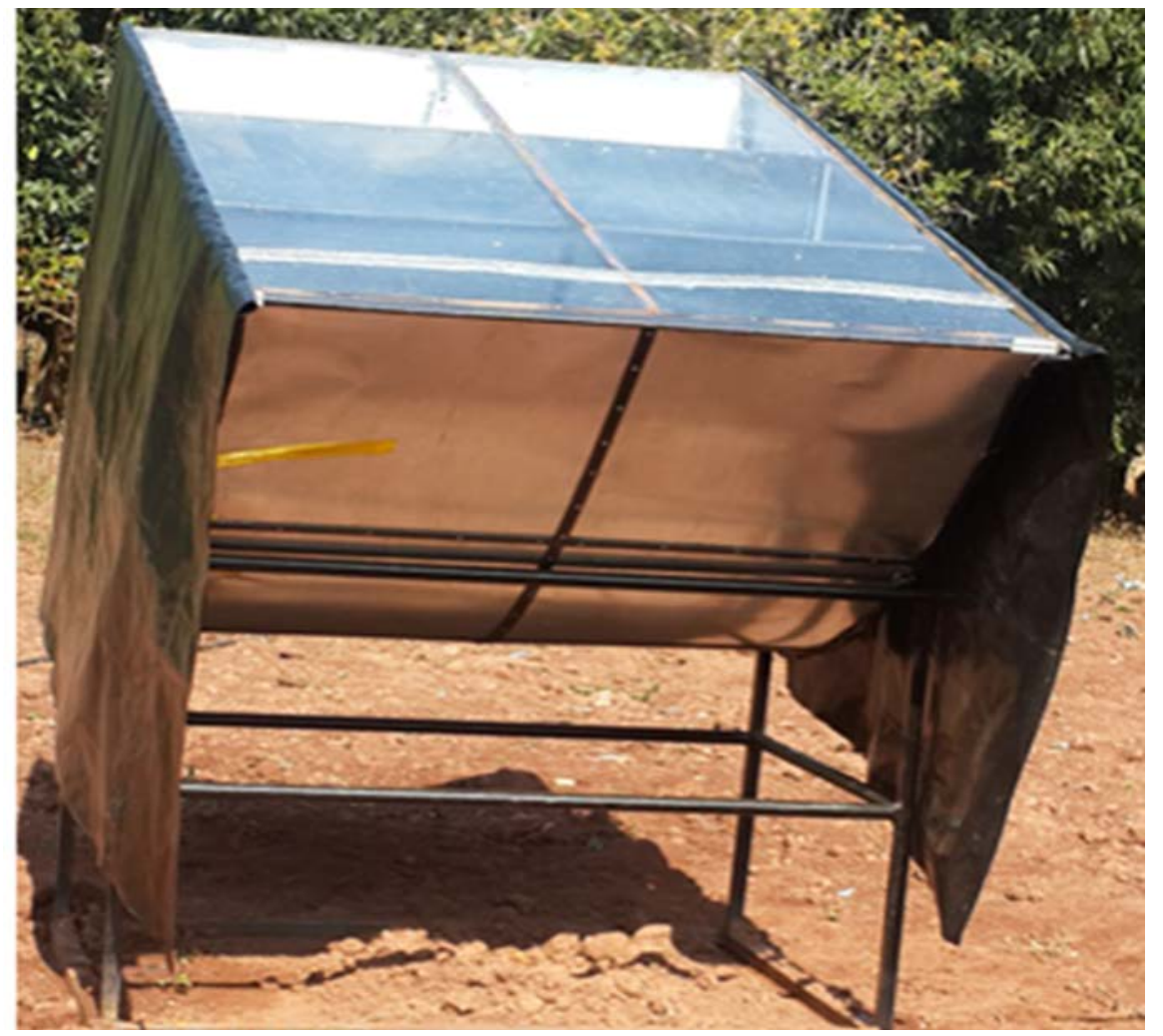

Figure 12. Completed installation of the four-wing $\mathrm{CPC}$ at the pilot trial site.

\subsection{Initial Trial Results of Sanitization of Waste Products}

Trials were made for testing the performance of the four wing solar concentrator using waste materials derived from urine diversion dry toilets. These consisted of composted fecal matter, fresh fecal matter, stored urine and grey water. Trials with liquid showed that temperature between 80 and 100 degree Celsius were 
generated during most hours of solar radiation. Because of the prolonged duration of sun ray trapping, this setup shows increased performance in terms of the duration during which concentrated heat is made available.

Trials for sanitization of composted as well as fresh fecal matter showed that the solid materials could attain temperature over 70 degree Celsius over a prolonged period of time. Further trial of the microbiological quality of these sanitized fecal matter products showed that the fecal coliform count was non-detectable in both samples. The concentrators were therefore, effective in killing of pathogens in a relatively short period of time. The only problem with the application of this set up for sanitization of composted or fresh fecal matter would be the limited volume available on the concentrator. For this reason it might be preferable to use another setup if the fecal matter volume to be sanitized is large.

Attempts were also made to concentrate urine after the ammonia from the urine was precipitated using dolomitic limeused as source of magnesium for the struvite precipitation. The procedure consisted of applying the urine on the absorber followed by the addition of proportional amount of dolomitic lime. While ammonia precipitates as struvite the excess ammonia and water evaporates and a black residue is left as concentrated products of the urine. In this way it is suggested that concentration of nutrients from urine can be made by combining struvite precipitation with solar concentration.

Because of the added flexibility of the design for tracking sun ray, the setup of the four-wing concentrator enabled concentrating rays throughout the day although the intensity of ray during early morning and late afternoons tend to be low in the southern hemisphere where Swaziland is located. In addition, because of the much tilted winter solstice positions for Swaziland, which is located at the lower latitude of the southern hemisphere, it is necessary to adjust the orientation of the solar concentrator during the different seasons. Figure 13 shows the

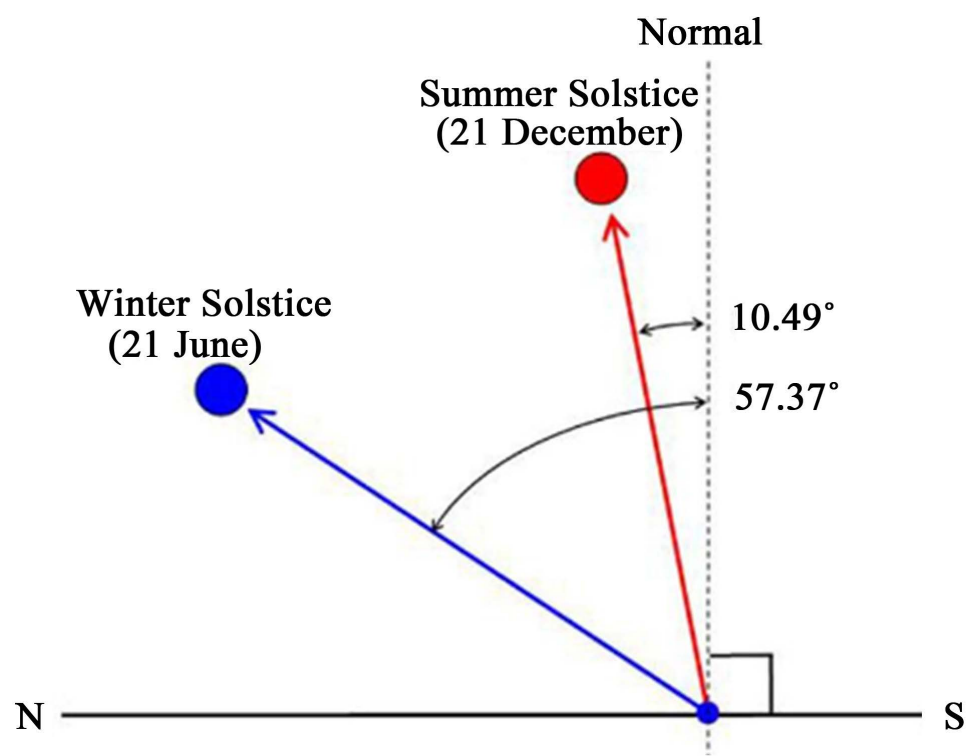

Figure 13. The different angles of incidence of sun ray during winter and summer in Swaziland. 
angle of incidence of sun ray for Swaziland during the different seasons. It can be seen that the angle of incidence is quite large during the winter season. Regular tracking might be necessary, therefore, especially in winter season because of the fact that the sun ray is at steeper angle of incidence. This position while giving less intensity of radiation requires tracking of the sun during the day.

\section{Conclusions}

The use of renewable energy resources such as the energy available from solar radiation is an environmental friendly approach that has been shown to have wider scope of application. It is the source of energy that is available at point of use with no visible cost associated except that of trapping it to usable level. Solar concentrators have long history of use. Since the 1960s, the application of nonimaging concentrators such as the compound parabolic concentrators has been in wider use for various purposes.

A modification of the compound parabolic concentrator in which the normally truncated portions of the parabola are included to set up a four-wing reflector has been studied in this research. Two alternative configurations were available which, in terms of the absorber configuration, consist of $\mathrm{V}$-shaped and flat-plate absorbers. The modified design increased the width of the aperture as well as the concentration ratio. In addition the period of time during which the sun ray can be trapped is increased.

The proposed four-wing CPC design is considered a hybrid of imaging and non-imaging concentrator because, for the part of the day in the early morning and late afternoon, one wing portion of the parabola acts as imaging concentrator provided the sun ray can be tracked. During mid-day the internal wings act as non-imaging concentrators. This design offers flexibility in terms of the use of concentrators enabling trapping of sun rays during the long hours of the day. The wider angle of acceptance also enables collection of as much of the diffused ray as possible similar to the asymmetric, truncated compound parabolic concentrators.

Pilot trials of the performance of the four-wing concentrators by using waste products derived from urine diversion dry toilets showed satisfactory results in terms of the intended use. High temperatures required for inactivation of pathogens reaching up to 90 degree Celsius were generated for liquid wastes such as grey water and urine. For solid wastes such as fresh and composted fecal matters, recorded temperatures of over 70 degree Celsius were reached. In addition, microbiological tests for coliforms confirmed the absence fecal coliforms for both composted and fresh fecal matter after they were exposed to high temperature using this solar concentrator set up. The set up was also tried for concentrating nutrients from urine combined with struvite precipitation.

The research set up showed useful results for the sanitization of liquid waste those of solid waste products of limited volume. Improvement of the materials and of heat preservation can be made further since this set up was constructed using locally available materials for reflector and absorber as well as for heat preservation. 


\section{Acknowledgements}

The financial support for this research was partly provided by the Government of Finland through the Mbabane Dry Sanitation and Waste Management Project. The authors are grateful for the financial assistance provided for this research in this regard.

\section{References}

[1] Mansi, G.S., Shah, P.K. and India, M.P. (2013) Design and Development of Compound Parabolic Concentrating Solar Collector with Flat Plate Absorber. International Journal of Innovative Research in Science, Engineering and Technology, 2.

[2] Sanchez, M., Martinez, I.G., Rincon, E.A. and Duran, M.A. (2014) Design and Thermal-Optic Analysis of an Ultra-Solar Concentrator. Energy Procedia, 57, 311 320.

[3] Winston, R. (1974) Principles of Solar Concentrator of a Novel Design. Solar Energy, 16, 89-95.

[4] Baranov, V.K. (1965) Opt. Mekh. Prom. 6, 1-5. A Paper in Russian That Introduces Certain Properties of CPCs.

[5] Baranov, V.K. (1967) Device for Restricting in One Plane the Angular Aperture of a Pencil of Rays from a Light Source. Russian Certificate of Authorship 200530. (In Russian)

[6] Baranov, V.K., and Melnikov, G.K. (1966) Study of the Illumination Characteristics of Hollow Focons. Soviet Journal of Optical Technology, 33, 408-411.

[7] Ploke, M. (1967) Lichtführungseinrichtungen mit starker Konzentrationswirkung. Optik, 25, 31-43.

[8] Hinterberger, H. and Winston, R. (1966) Efficient Light Coupler for Threshold Cerenkov Counters. Review of Scientific Instruments, 37, 1094-1095. https://doi.org/10.1063/1.1720428

[9] Hinterberger, H. and Winston, R. (1966) Gas Counter with Optimized Light-Collecting Efficiency. Instrumentation for High Energy Physics, Proceedings of the 1966 International Conference, Stanford, 9-10 September 1966, 205-206.

[10] Winston, R. (1970) Light Collection within the Framework of Geometrical Optics. Journal of the Optical Society of America, 60, 245-247. https://doi.org/10.1364/JOSA.60.000245

[11] Smestad, G., Ries H., Winston, R. and Yablonovitch, E. (1990) The Thermodynamic Limit of Light Concentrators. Solar Energy Materials, 21, 99-111.

[12] Winston, R. and Hinterberger, H. (1975) Principles of Cylindrical Concentrators for Solar Energy. Solar Energy, 17, 255-258.

[13] Harper, D.A., Hildebrand, R.H., Pernlic, R. and Platt, S.R. (1976) Heat Trap: An Optimized Far Infrared Field Optics System. Applied Optics, 15, 53-60. https://doi.org/10.1364/AO.15.000053

[14] Rabl, A. (1976) Comparison of Solar Concentrators. Solar Energy, 18, 93.

[15] Rabl, A. (1976) Optical and Thermal Properties of Compound Parabolic Concentrator. Solar Energy, 18, 497-511.

[16] Winston, R., Juan, C. and Benítez, P. (2005) Non-Imaging Optics. Elsevier Academic Press, Cambridge, 497 p.

[17] Rabl, A. (1985) Active Solar Collectors and Their Applications. Oxford University Press, New York, 503 p. 
[18] Mallick, T.K. (2003) Optics and Heat Transfer for Asymmetric Compound Parabolic Photovoltaic Concentrators for Building Integrated Photovoltaics. PhD Thesis, Faculty of Engineering, University of Exeter, Exeter.

[19] Rönnelid, M. and Karlsson, B. (2003) Optimised Truncation of a Wide Acceptance Angle CPC. Proceedings of ISES Solar World Congress, Gothenburg, 14-19 June 2003.

[20] Nilson, J. (2005) Optical Design and Characterization of Solar Concentrators for Photovoltaics. Report EBD-T-05/6, Division of Energy and Building Design, Department of Architecture and Built Environment Lund University, Faculty of Engineering LTH.

[21] APHA (1989) Standard Methods for the Examination of Water and Wastewater. 17th Edition, American Public Health Association, Washington DC, 268 p.

[22] United States Department of Agriculture (USDA) (2014) Most Probable Number Procedure and Tables. Food Safety and Inspection Service, Office of Public Health Science, Georgia.

\section{Submit or recommend next manuscript to SCIRP and we will provide best service for you:}

Accepting pre-submission inquiries through Email, Facebook, LinkedIn, Twitter, etc. A wide selection of journals (inclusive of 9 subjects, more than 200 journals)

Providing 24-hour high-quality service

User-friendly online submission system

Fair and swift peer-review system

Efficient typesetting and proofreading procedure

Display of the result of downloads and visits, as well as the number of cited articles

Maximum dissemination of your research work

Submit your manuscript at: http://papersubmission.scirp.org/

Or contact jpee@scirp.org 